\title{
El conmutador inteligente de potencia y la sub-medición por circuito como herramientas para la gestión energética residencial
}

\section{The intelligent power switch and the sub-measurement circuit as tools for residential energy management}

\author{
Luis Fernando Rueda-Vásquez ${ }^{1}$, Jaime Guillermo Barrero-Pérez² ${ }^{2}$ Cesar Duarte $^{3}$ \\ ${ }^{1}$ Grupo de Investigación en control, electrónica, modelado y simulación - CEMOS, Escuela de Ingeniería Eléctrica, Electrónica y \\ de Telecomunicaciones, Universidad Industrial de Santander, Colombia. Email: luisfruedav@gmail.com \\ ${ }^{2}$ Grupo de Investigación en control, electrónica, modelado y simulación - CEMOS, Escuela de Ingeniería Eléctrica, Electrónica y \\ de Telecomunicaciones, Universidad Industrial de Santander, Colombia. Email: jbarrero@uis.edu.co \\ ${ }^{3}$ Grupo de Investigación en Sistemas de Energía Eléctrica - GISEL, Escuela de Ingeniería Eléctrica, Electrónica y de \\ Telecomunicaciones, Universidad Industrial de Santander, Colombia. Email: cedagua@uis.edu.co
}

Recibido: Septiembre 30, 2016 Aceptado: Noviembre 30, 2016 Versión Final: Diciembre 30, 2016

\section{RESUMEN}

Este documento presenta el concepto de un hogar inteligente, sus componentes y sus aplicaciones desde el punto de vista de ahorro y gestión energética, describiendo las características y el funcionamiento de la sub-medición por circuito y el conmutador inteligente de potencia, al igual que su integración con el hogar inteligente. Asimismo, se muestra la reglamentación y normativa colombiana que promueve el uso racional y eficiente de energía, la utilización de fuentes no convencionales de energía eléctrica y los primeros pasos hacia la implementación de las redes inteligentes en Colombia. De igual forma, se presenta la topología e implementación de un sistema de distribución residencial, el cual permite controlar el flujo de energía entre diferentes fuentes de energía y las cargas de una vivienda. Finalmente, se analiza el comportamiento de la tensión en las cargas ante diferentes escenarios de conexión, observando la importancia de realizar adecuadamente las conmutaciones en el IPS.

PALABRAS CLAVE: Hogar inteligente, Conmutador Inteligente de Potencia, Sub-medición, Fuentes renovables.

\begin{abstract}
This paper introduces the concept of Smart Home, its components and applications in terms of saving and energy management, describing the characteristics and the operation of the sub-metering by circuit and the intelligent power switch, as well as its integration with the smart home. In addition to that, the regulation and Colombian legislation that promotes the rational and efficient use of energy, the use of non-conventional sources of electricity and the first steps towards the implementation of smart grids in Colombia, are exposed. This research presents the topology and the implementation of a residential distribution system, which allows controlling the energy flow through different energy sources and housing loads. Finally, the behavior of voltage in the loads in different connection scenarios is analyzed, stressing the importance of the appropriate realization of commutations at the IPS.
\end{abstract}

KEYWORDS: Smart Home, Intelligent Power Switch, Sub-metering, Renowable sources.

\section{INTRODUCCIÓN}

Evidencia científica internacional señala que desde 1750 el planeta está experimentando un calentamiento neto, y que durante el presente siglo continuará calentándose a

Consecuencia de las emisiones de gases de efecto invernadero (GEI) producidos por la acción humana, en particular la procedente del consumo de petróleo y carbón. Este es, sin duda, el problema más grave en el campo ambiental, y según muchas autoridades, la mayor amenaza global en términos absolutos [1].

En el aspecto energético el cambio climático presenta desafíos cada vez mayores para su producción y transmisión. El aumento progresivo de la temperatura, el

Este artículo puede compartirse bajo la licencia CC BY-ND 4.0 y se referencia usando el siguiente formato: L. Rueda, J. Barrero, C. Duarte. El conmutador inteligente de potencia y la sub-medición por circuito como herramientas para la gestión energética residencial," UIS Ingenierías, vol. 16, no. 1, pp. 35-46, Enero-Junio 2017. 
creciente número y severidad de los fenómenos meteorológicos extremos y el cambio de los patrones de precipitación afectarán la producción y el suministro de energía [2]. Así mismo, el suministro de combustibles fósiles y la generación y transmisión de energía térmica e hidroeléctrica, también se verán afectados.

De esta manera, la expectativa de reducción de los impactos globales ha propiciado alternativas como: la generación distribuida, la respuesta a la demanda, la tarifa diferencial, las microredes de energía y los sistemas de gestión energética residencial, entre otros. Las nuevas tecnologías convergen bajo el concepto de las redes inteligentes (Smart Grids), los cuales tienen el potencial para lograr un nuevo nivel de gestión energética [3], [4].

Considerando la creciente demanda de electrodomésticos, dispositivos móviles de entretenimiento digital y comunicaciones, el sector residencial es un sector con vasto potencial de ahorro energético, haciéndose necesario el uso de electrodomésticos y dispositivos inteligentes con capacidad de ahorrar energía, reducir costos e incrementar la confiabilidad y robustez de la red.

La integración de fuentes no convencionales de energía ha incrementado rápidamente. Sin embargo, estas fuentes alternativas no cuentan con la capacidad de proveer energía de forma constante, presentando fluctuaciones en la potencia de salida, la frecuencia de la red y el valor eficaz de la tensión [5], [6]. Como alternativa para superar esa limitante de las fuentes renovables, en 1998, Toyoda propuso el concepto de una red de energía eléctrica abierta (Open Electric Energy Network, OEEN), donde el flujo de potencia es controlado por múltiples Electric Energy Routers [7]. Este Power Router permite que diferentes tipos de fuentes de energía que pueden estar dispersas sean integradas al mercado de energía eléctrica sin causar inconvenientes a los servicios de energía existentes o a los consumidores [8].

De igual manera, diversos estudios han mostrado que sólo con brindar a los usuarios el consumo desagregado de la energía y proveer retroalimentación frente a su gasto energético se logra reducir el consumo hasta en un $18 \%$, en especial debido a que la información suministrada al usuario final sobre los patrones de consumo propicia la gestión individual de la demanda [9], [10]. Es por esto, que la sub-medición por circuito es una herramienta importante para el uso racional y eficiente de la energía eléctrica.

Este artículo presenta el conmutador inteligente de potencia y la sub-medición por circuito como herramientas para la gestión energética residencial, para esto, se realiza una revisión de los antecedentes y el estado del arte relacionado con la temática. A continuación, se presenta la topología de un sistema de distribución de potencia residencial, por medio del cual es posible controlar el flujo de energía entre las diferentes fuentes de energía y las cargas de una vivienda. Igualmente, se describe la implementación del sistema propuesto y se analizan los resultados de los experimentos realizados.

El documento está organizado de la siguiente manera. La Sección 2 presenta el concepto de hogar inteligente, sus características y funcionalidades. Posteriormente, la Sección 3 muestra la reglamentación y normativa colombiana relacionada con la gestión energética. A continuación, en las Secciones 4 y 5 respectivamente, se describen los tipos de router de energía eléctrica y el conmutador inteligente de potencia. De la misma manera, la Sección 6 expone la sub-medición por circuito y en la Sección 7 la describe la metodología utilizada para la investigación. Finalmente, la Sección 8 presenta los experimentos y resultados obtenidos y la Sección 9 las conclusiones del trabajo realizado.

\section{HOGARES INTELIGENTES}

Un hogar inteligente (Smart Home) es la integración de diferentes servicios y componentes dentro de una unidad residencial utilizando sistemas de comunicación, capaces de soportar operaciones seguras y económicas, además de brindar confort y gestión de la carga eléctrica según las preferencias del usuario. Un hogar inteligente en un principio fue concebido como una tecnología para controlar sistemas ambientales de iluminación y temperatura. Actualmente esta tecnología también permite la monitorización de las actividades al interior de la casa, dando la posibilidad de una operación individualizada según las costumbres y las preferencias del usuario previamente censadas. Este nivel de inteligencia, flexibilidad y funcionalidad tienen una vasta variedad de aplicaciones [11], [12].

\subsection{Aplicaciones}

La infraestructura de comunicación y control de un hogar inteligente permite una gran variedad de aplicaciones de diferentes requerimientos y beneficios.

A continuación, se muestran tres categorías de aplicaciones:

\subsubsection{Protección y seguridad.}

Incluye sistemas de alarma y monitorización, operaciones seguras de equipos técnicos o asistencia médica en caso de emergencia a usuarios con limitaciones. 


\subsubsection{Confort, comunicación y entretenimiento.}

Sistemas programables de control de iluminación, persianas, puertas y diferentes clases de dispositivos residenciales, dispositivos de entretenimiento como radio, televisión, teatro en casa y dispositivos wifi e integración de los diferentes protocolos de comunicación dentro de la red de área doméstica.

\subsubsection{Gestión energética.}

Este tipo de aplicaciones se componen de un conjunto de sistemas que permiten realizar control eficiente de cargas eléctricas y sistemas de climatización de edificaciones (Heat Ventilation and Air Condiotinning, HVAC), monitorización y control del consumo desagregado de energía eléctrica, la incorporación del vehículo eléctrico, la integración de fuentes no convencionales de energía eléctrica y brindar servicios complementarios al usuario y a la empresa prestadora del servicio..

\subsection{Componentes}

En la Figura 1 son presentados los componentes típicos de un hogar inteligente para aplicaciones de gestión energética. Se puede observar la presencia de fuentes no convencionales de energía, el vehículo eléctrico, la infraestructura de medición avanzada (Advanced Metering Infrastructure, AMI), la red de área doméstica (Home Area Network, HAN), el concentrador y los electrodomésticos inteligentes. Adicionalmente, el hogar cuenta con conectividad a internet, haciendo posible mantener una comunicación bidireccional con la empresa electrificadora y con el usuario, permitiéndole a este último monitorizar y controlar su hogar de forma remoto por medio de un computador o dispositivo móvil.

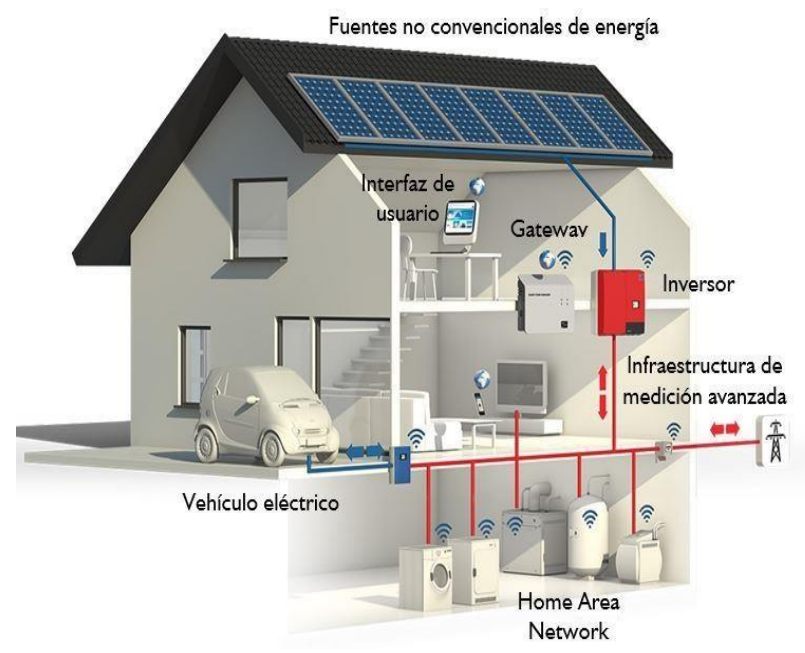

Figura 1. Componentes de la Smart home. Fuente. [13].

De igual manera, en la Figura 2 se muestra la estructura de un hogar inteligente de forma esquemática. Se observa que la vivienda esta equipado con dispositivos inteligentes, una red de comunicación doméstica que es capaz de mantener conectados los dispositivos, un concentrador que recibe toda la información y gestiona las diversas funcionalidades del sistema, y el Gateway, el cual es una pasarela entre el hogar y el usuario a través de Internet [14].

\section{MARCO LEGAL Y REGULACIÓN COLOMBIANA}

La gestión energética residencial en Colombia está en un nivel reglamentario bajo para la introducción de tecnologías como las redes inteligentes, la infraestructura de medición avanzada y los sistemas de gestión energética residenciales (Home Energy Management System - HEMS). Sin embargo, en los últimos años se ha venido evolucionando en este contexto. A continuación, se exponen los aspectos más relevantes de la reglamentación y promulgación en materia de eficiencia energética por parte de las entidades gubernamentalesde manera apropiada un artículo científico original.

\subsection{Ley 1715 de 2014}

Promueve el desarrollo y la utilización de las fuentes no convencionales de energía, principalmente aquellas de carácter renovable, en el sistema energético nacional, mediante su integración al mercado eléctrico, su participación en las zonas no interconectadas y en otros usos energéticos como medio necesario para el desarrollo económico sostenible, la reducción de emisiones de gases de efecto invernadero y la seguridad del abastecimiento energético.

Con esta ley se busca establecer el marco legal y los instrumentos para la promoción del aprovechamiento de las fuentes no convencionales de energía, lo mismo que para el fomento de la inversión, investigación y desarrollo de tecnologías limpias para producción de energía, la eficiencia energética y la respuesta de la demanda, en el marco de la política energética nacional [15].

\subsection{Decreto 1285 y resolución 549 de 2015}

Encaminados al mejoramiento de la calidad de vida de los habitantes y el ejercicio de actuaciones con responsabilidad ambiental y social, el 10 de julio de 2015 el Ministerio de Vivienda, Ciudad y Territorio (MVCT) anunció la adopción de la primera reglamentación de índole nacional que fomenta la construcción sostenible. Se trata de la Resolución No. 549 y el Decreto 1285 de 2015 del MVCT [16] mediante la cual se adopta la Guía para el ahorro de agua y energía en edificaciones. 
El objetivo de esta guía es proporcionar una herramienta para la implementación de estrategias de construcción sostenible para ser aplicadas en los municipios de todo el país. La guía pretende promover eficiencia energética y conservación del agua durante el uso de las edificaciones.
Para esto, establece la proporción del consumo de agua y energía que se pretende disminuir mediante la implementación de medidas activas y pasivas en el diseño de las edificaciones [17].

Tabla1. Contexto legal, normativo y reglamentario colombiano encaminado a la gestión, eficiencia y uso racial de la energía eléctrica.

\begin{tabular}{|c|c|}
\hline Nombre & Descripción \\
\hline $\begin{array}{l}\text { Ley } 697 \text { de } \\
2001\end{array}$ & $\begin{array}{l}\text { Esta ley designa los incentivos y las responsabilidades dentro del desarrollo del Programa de } \\
\text { Uso Racional y Eficiente de la Energía y demás Formas de Energía No Convencionales } \\
\text { (PROURE). Este programa tiene como objetivo aplicar gradualmente programas de ahorro } \\
\text { energético y niveles mínimos de eficiencia, los cuales son preestablecidos por la ley para todo } \\
\text { el sistema energético [18], [19]. }\end{array}$ \\
\hline $\begin{array}{l}\text { Decreto } 3683 \\
\text { de } 2003\end{array}$ & $\begin{array}{l}\text { Reglamenta el uso racional y eficiente de la energía, de tal manera que se tenga la mayor } \\
\text { eficiencia energética para asegurar el abastecimiento energético pleno y oportuno, la } \\
\text { competitividad del mercado energético colombiano, la protección al consumidor y la } \\
\text { promoción de fuentes no convencionales de energía, dentro del marco del desarrollo sostenible } \\
\text { y respetando la normatividad vigente sobre medio ambiente y los recursos naturales renovables } \\
\text { [20]. }\end{array}$ \\
\hline $\begin{array}{l}\text { Decreto } 2501 \\
\quad \text { de } 2007\end{array}$ & $\begin{array}{l}\text { Busca propiciar el uso racional y eficiente de energía eléctrica en [21]: } \\
\text { - Productos de uso doméstico. } \\
\text { - Edificaciones donde funcionen entidades públicas. } \\
\text { - Para viviendas de interés social. }\end{array}$ \\
\hline $\begin{array}{l}\text { Decreto } 3450 \\
\quad \text { de } 2008\end{array}$ & $\begin{array}{l}\text { Reglamenta el cambio de las fuentes de iluminación de baja eficacia por fuentes con mayor } \\
\text { eficacia lumínica disponibles en el mercado [22]. }\end{array}$ \\
\hline $\begin{array}{l}\text { Resolución } \\
180919 \text { de } \\
2010\end{array}$ & $\begin{array}{l}\text { Define unos subprogramas estratéticos, metas e indicadores para la aplicación gradual en toda } \\
\text { la cadena energética a fin de crear condiciones en el uso racional y eficiente de la energía y } \\
\text { aprovechamiento de las fuentes no convencionales de energía en Colombia [23]. }\end{array}$ \\
\hline $\begin{array}{l}\text { Programa } \\
\text { Colombiano de } \\
\text { Normalización, } \\
\text { Certificación y } \\
\text { Etiquetado de } \\
\text { Equipos de Uso }\end{array}$ & $\begin{array}{l}\text { Es una herramienta que pretende el óptimo aprovechamiento de los recursos naturales en toda } \\
\text { la cadena energética, como mecanismo práctico para el establecimiento de condiciones de } \\
\text { mercado que promuevan la penetración de tecnología energéticamente eficiente y como } \\
\text { estrategia para la generación de una cultura en uso racional y eficiente de la energía en la } \\
\text { sociedad colombiana [24]. }\end{array}$ \\
\hline $\begin{array}{l}\text { Norma ISO } \\
50001\end{array}$ & $\begin{array}{l}\text { Estándar internacional donde se establecen los requisitos para la adecuada gestión de la } \\
\text { energía, orientado a la aplicación en todo tipo de organizaciones y empresas. La norma } \\
\text { pretende promover planes de acción enfocados a aumentar la eficiencia energética, reducción } \\
\text { de costos y consumos de energía [25]. }\end{array}$ \\
\hline $\begin{array}{l}\text { Resolución } 038 \text { de } \\
2014 \\
\text { - Código de } \\
\text { medida }\end{array}$ & $\begin{array}{l}\text { Reglamenta la mínima clase de exactitud para los medidores de energía activa y el error } \\
\text { máximo permitido. Igualmente, dispone que todos los componentes del sistema de medición } \\
\text { deben contar con certificado de conformidad y por medio de la NTC } 4052 \text { establece las normas } \\
\text { de fabricación de los medidores de energía [26]. }\end{array}$ \\
\hline NTC 6079 & $\begin{array}{l}\text { Esta norma ha sido elaborada con el fin de establecer los requerimientos mínimos que deben } \\
\text { cumplir los sistemas AMI para su operación y gestión [27]. }\end{array}$ \\
\hline
\end{tabular}

Fuente:Elaboración propia. 


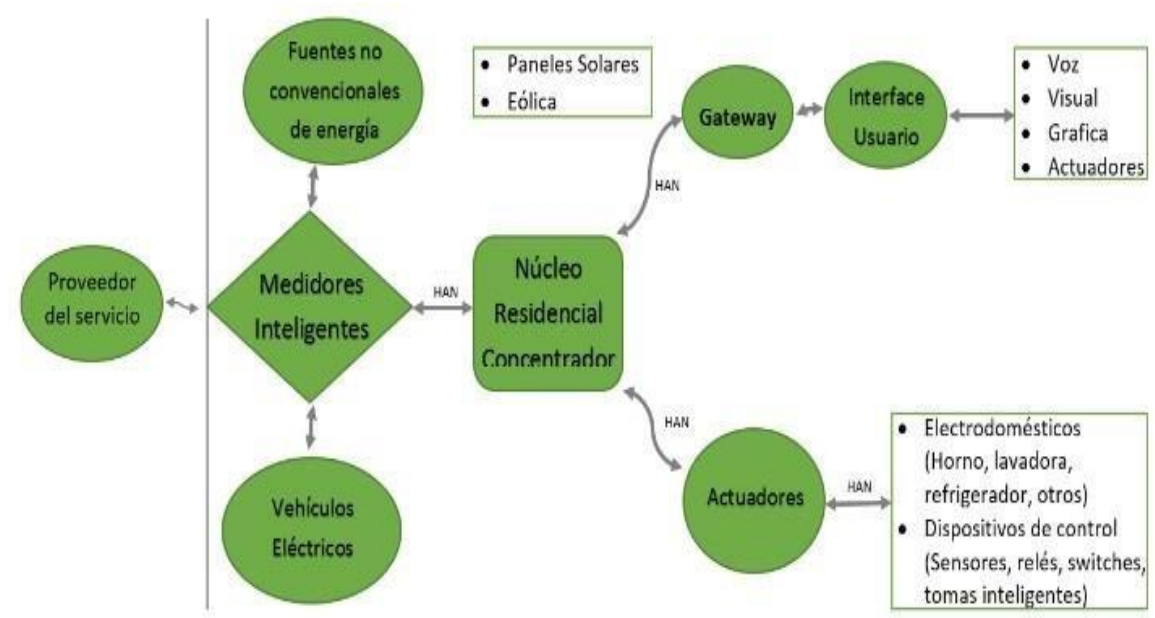

Figura 2. Diagrama esquemático de un hogar inteligente. Fuente. Elaboración propia.

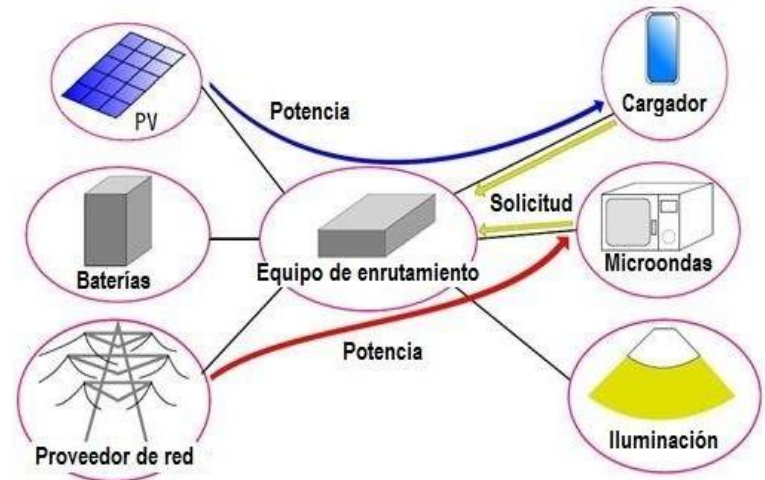

Figura 3. Red de distribución AC de un hogar. Fuente. [6].

\subsection{Otras reglamentaciones}

En la Tabla 1 se presentan otras normativas que han permitido la evolución del contexto legal, normativo y reglamentario colombiano encaminado a la gestión, eficiencia y uso racial de la energía eléctrica.

\section{ENRUTADOR DE ENERGÍA ELÉCTRICA}

La instalación de fuentes renovables de energía como los paneles fotovoltaicos, turbinas eólicas y/o celdas de combustible en conjunto con la red eléctrica comercial, produce electricidad de calidad variable en los hogares debido a las fluctuaciones que las fuentes no convencionales de energía eléctrica introducen a la red [6].

La calidad de la energía eléctrica implica un suministro de potencia eléctrica máxima disponible, además de presentar estabilidad de frecuencia y tensión [28]. En general, la energía generada por fuentes renovables es inferior en calidad a la energía comercial.
Actualmente, el uso de baterías secundarias es combinado con las fuentes de generación distribuida para compensar los cambios en la potencia eléctrica de salida, ajustando la calidad de la energía de salida a la de la de la red comercial por medio de inversores y conversores. Sin embargo, este método es ineficiente debido a que incrementa las pérdidas del sistema a causa de las múltiples conversiones [28]. Algunos equipos como los computadores portátiles y los dispositivos móviles son construidos con baterías internas, no siendo necesario que estos sean alimentados con energía de alta calidad. Por tanto, es razonable conectar una determinada fuente de energía eléctrica con ciertas cargas, dependiendo de las necesidades de cada una de estas [28].

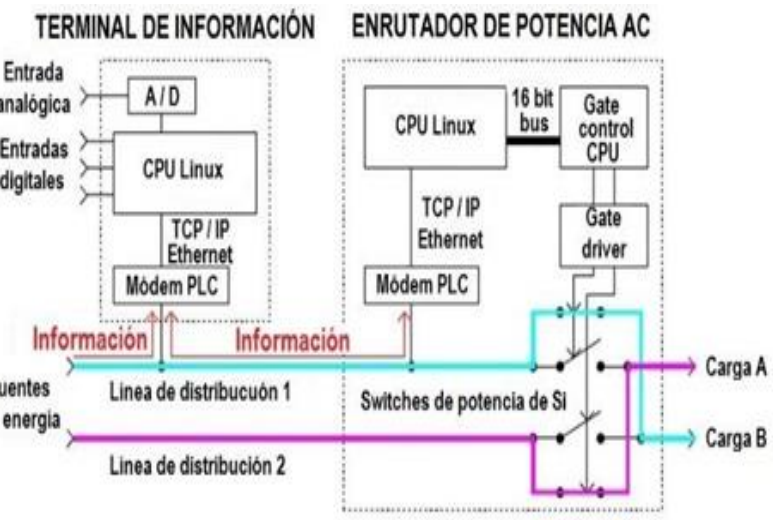

Figura 4. Esquemático del Enrutador de Potencia AC y su terminal de información. Fuente. [8].

La Figura 3 muestra el mapa conceptual de la red de distribución AC de un hogar. En este, las diferentes fuentes de energía eléctrica externas o propias y los circuitos eléctricos internos del hogar son conectados al 
equipo de enrutamiento (AC Power Router), de esta manera, cuando el usuario desea utilizar un aparatoeléctrico, este envía una solicitud de suministro al enrutador, correspondiente a la cantidad de potencia necesaria, la calidad de potencia requerida, el número de identificación del aparato, la prioridad de las solicitudes, y así sucesivamente.

Para lograr esto, el sistema está compuesto por una terminal de información y por el enrutador de potencia (Figura 4). De esta manera, el enrutador recibe la información por medio de un protocolo de comunicación (por ejemplo Power Line Communications - PLC) y posteriormente selecciona una fuente de energía eléctrica adecuada a las características de la carga [6]. Esto implica que el sistema esta administrado de forma centralizada, permitiendo que las cargas que incorporan baterías tengan un suministro de potencia relativamente de baja calidad. Mientras que, por el contrario, la energía eléctrica comercial de alta calidad se suministra preferiblemente a cargas o equipos importantes, tales como instrumentos médicos, que no tienen la tolerancia a la caída de tensión momentánea. Así, el equipo de enrutamiento decide utilizar fuentes de energía renovables tanto como sea posible [6].

\section{CONMUTADOR INTELIGENTE DE POTENCIA}

En [29] es presentado el concepto de la red eléctrica del futuro; proponiendo una innovadora arquitectura de energía eléctrica que integra funcionalidades tanto de Internet como de las micro-redes. El componente principal de esta arquitectura es un conmutador inteligente de potencia (Intelligent Power Switch, IPS) que combina las capacidades de un enrutador de Internet con la conversión de energía eléctrica y la protección de equipos [29].

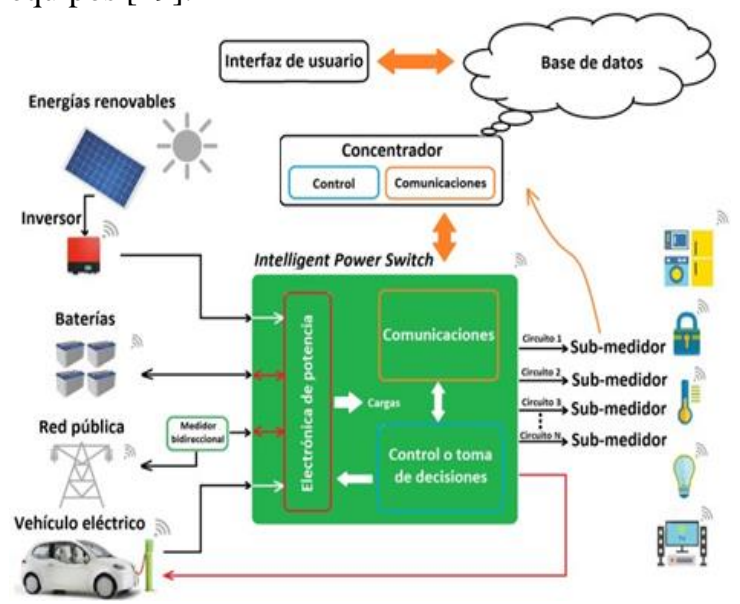

Figura 5. Integración del conmutador inteligente de potencia y la sub-medición por circuito. Fuente. Elaboración. propia.
La Figura 5 se presenta un sistema de gestión que integra el conmutador inteligente de potencia y la sub- medición por circuito. Se observa que el IPS es un dispositivo que puede re-direccionar el flujo de energía eléctrica de un puerto a otro, y respecto a su funcionamiento, actúa como un enrutador de energía eléctrica por medio de su etapa de potencia. Además, está equipado con una etapa de control, otra de comunicación inalámbrica y/o cableada y como se muestra en la Figura 5, es posible conectarlo con diferentes fuentes de energía (incluidas las fuentes renovables), electrodomésticos inteligentes, almacenamiento de energía, medidores inteligentes y también otros IPS.

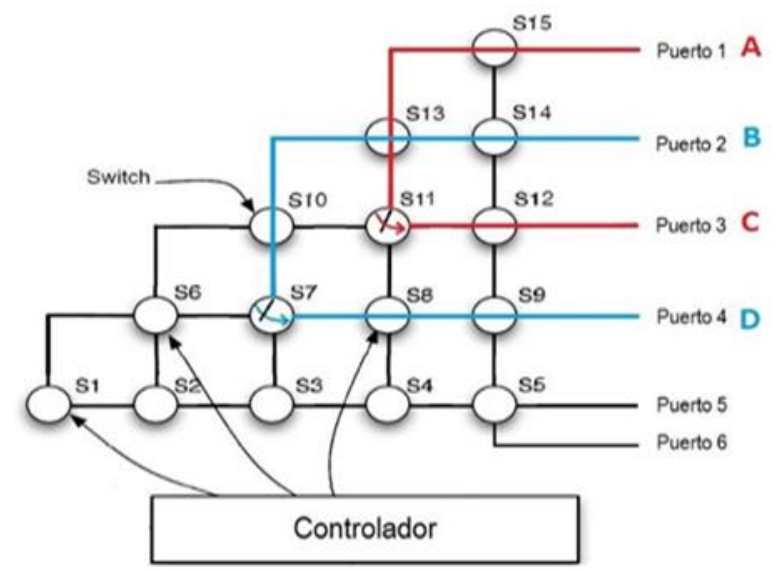

Figura 6. Esquema interno del conmutador inteligente de potencia. Fuente. [30].

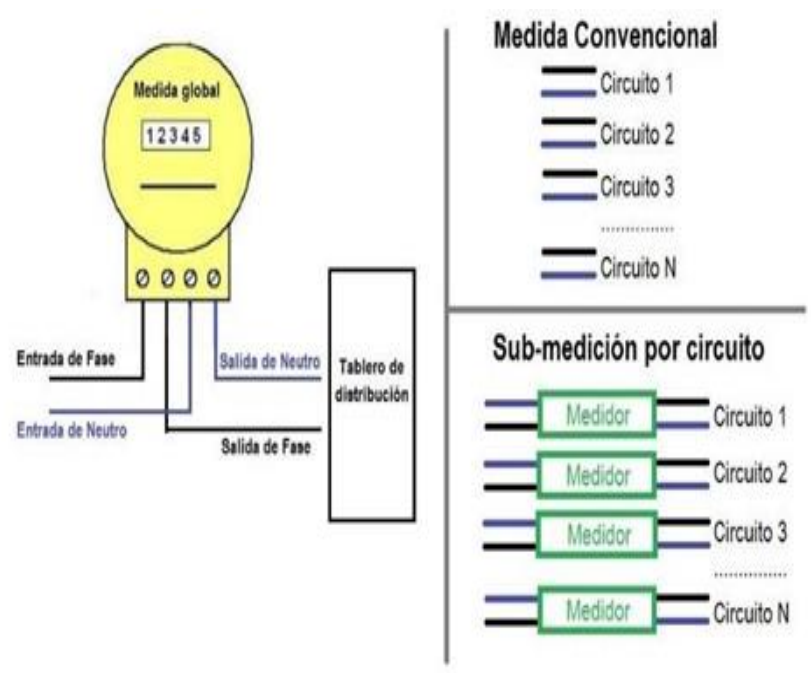

Figura 7. Esquema interno del conmutador inteligente de potencia. Fuente. Elaboración propia

De esta manera, un IPS permite crear una red eléctrica distribuida y escalable, controlando eficientemente la interconexión de sus componentes [16]. 
El propósito de un IPS es conmutar la energía eléctrica de un puerto a otro, es decir que cualquier componente conectado al IPS puede estar conectado otro, o al mismo tiempo múltiples puertos pueden ser conectados juntos [30]. Esto significa que, si se tienen dos fuentes de energía eléctrica, A y B, y dos cargas eléctricas, C y D, A puede proveer energía eléctrica a $\mathrm{C}$ y $\mathrm{B}$ puede suministrar energía eléctrica a D paralelamente. Para lograr este objetivo, el IPS usa el diseño presentado en la Figura 6, en el cual son utilizados quince interruptores para controlar seis puertos [30].

En caso de ser requeridos más puertos, la cantidad de switches es calculada por la Ecuación 1, dónde NS es el número de puertos y NS el número de switches necesarios para el IPS [30].

$$
N_{S}=\underline{\underline{N} \underline{k}\left(N_{\underline{p}}-1\right)}
$$

\section{SUB-MEDICIÓN POR CIRCUITO}

Con estos sistemas se busca brindar información más detallada y en tiempo real del consumo de energía eléctrica de cada sector de la vivienda, lo cual en conjunto con el conmutador inteligente de potencia (Figura 5), hacen posible la implementación de programas de gestión energética y uso racional de la energía que permitan la reducción del consumo y los costos económicos.

Igualmente, dentro de la guía para el ahorro de agua y energía en edificaciones presentada en [16] y [17], la submedición por circuito es planteada como una medida pasiva que busca mejorar la eficiencia de las edificaciones, ayudando al usuario a entender la distribución del consumo de energía y proveer suficiente información sobre cual comportamiento de acción/corrección de conservación de energía debe aplicarse.

En la Figura 7 se observa la diferencia entre una medida convencional y la realizada por medio de la sub-medición por circuito. Para esto, la recolección de los datos se realiza mediante dispositivos de sub-medida independientes, adicionales a los provistos por las compañias de distribución de energía, uno por circuito ramal [31]. Estos dispositivos electrónicos tienen además de su módulo de medición de energía eléctrica, un módulo de comunicación que perite su monitorización y control desde el concentrador de la Smart Home.

\section{METODOLOGÍA}

Para el desarrollo del artículo se utiliza la topología del sistema de distribución residencial presentada en la
Figura 8. Se observa la presencia de un conmutador inteligente de potencia de cuatro puertos, en donde dos de estos puertos son conectados a fuentes de tensión independientes (la red comercial y una segunda fuente que puede ser un sistema fotovoltaíco u otro) de las cuales es posible conocer su disponibilidad de potencia y la potencia suministrada o recibida de cada una de estas por medio de medidores inteligentes y/o protocolos de comuicación inalambrica o cableada.

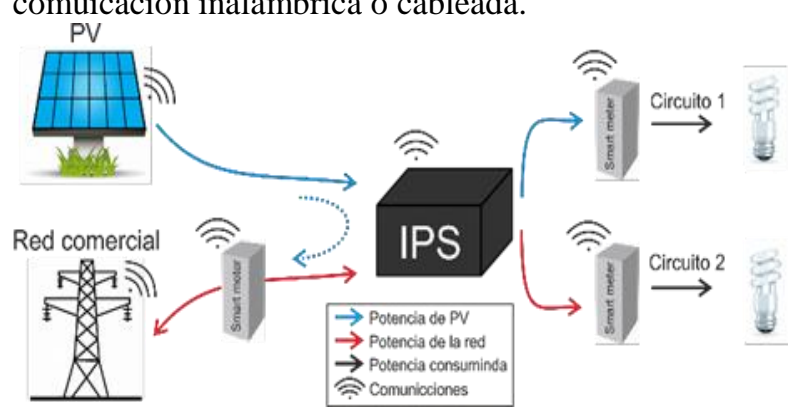

Figura 8. Topología del sistema de distribución residencial para la integración del conmutador inteligente de potencia y la sub-medición por circuito. Fuente. Elaboración propia.

Adicionalmente, los dos puertos restantes del IPS son conectados a dos circuitos ramales, los cuales cuentan con un medidor inteligente y una carga resistiva para cada uno. Es importante resaltar que la comunicación de los medidores inteligentes con el IPS se realizada por medio del protocolo Wifi, incorporando el concepto del internet de las cosas (Internet of Things, IoT).

\subsection{Implementación}

Con base en la topología propuesta, en la Figura 9 se presenta el diagrama esquemático de la implementación del IPS de cuatro puertos realizado. De manera general, el IPS puede ser dividido en dos componentes principales: el primero es el sistema embebido, que en este caso es la tarjeta de desarrollo Raspberry Pi, y un segundo componente, que es la etapa de electrónica de potencia o switches de potencia.

\subsubsection{Sistema embebido.}

Es el encargado de brindar al IPS las funcionalidades de comunicación y control. Para esto, es posible hacer uso de protocolos de comunicación inalámbrica como el Bluetooth o el Wifi, o protocolos cableados como el UART, el I2C, el SPI o el USB para intercambiar información con las fuentes de energía, las cargas, otro IPS, la red comercial y los usuarios. De esta manera, es posible conocer la información relacionada con la demanda de las cargas, la capacidad de energía que pueden suministras las fuentes y la tarifa de la energía eléctrica. Esta información es importante ya que son los parámetros de entrada para el algoritmo de control encargado de la apertura o cierre de los switches de la 
etapa de potencia. Adicionalmente, es posible enviar información al proveedor del servicio y a los usuarios por medio de la conexión a internet.

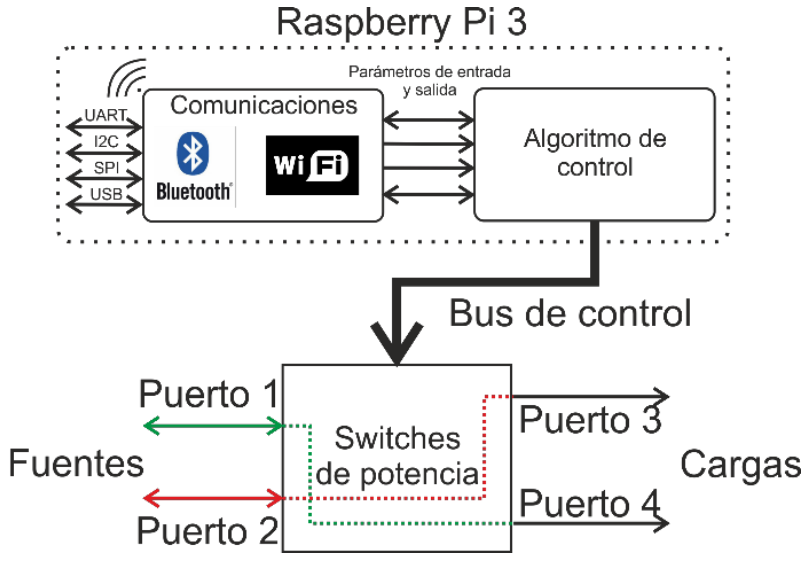

Figura 9. Esquema interno del conmutador inteligente de potencia. Fuente. Elaboración propia.

\subsubsection{Electrónica de potencia.}

$\mathrm{Su}$ función es permitir la conmutación de la energía eléctrica de un puerto a otro, es decir que cualquier componente conectado al IPS puede estar conectado otro, o al mismo tiempo múltiples puertos pueden ser conectados entre si.

\section{EXPERIMENTOS Y RESULTADOS}

Se presentan cuatro escenarios de conexión que permiten evidenciar el funcionamiento del sistema propuesto. Para esto, se analiza el comportamiento de la tensión en las cargas, teniendo como referencia el patrón de conmutación utilizado para cada uno de las pruebas.

Para la realización de los experimentos son utilizadas dos fuentes de tensión de $120 \mathrm{~V}, 60 \mathrm{~Hz}$ desfasadas $120^{\circ}$ entre sí (Figura 10) y dos cargas resistivas (luminarias halógenas) con un consumo de potencia de $70 \mathrm{~W}$ cada una.

Teniendo en cuenta que para la etapa de electrónica de potencia del IPS se utilizaron alternistores, se debe asegurar que al momento de realizar una conmutación entre puertos, los switches deben desconectarse de forma natural (señal de bajo en el gate y cruce por cero de la corriente), ya que de no ser así, se puede presentar la circulación de corrientes muy elevadas (de corto circuito) que pueden acortar la vida útil del IPS o su destrucción. Esto puede suceder cuando se conectan fuentes de tensión desfasadas entre sí o por el manejo de cargas RL con diferente factor de potencia.

Para evitar esta situación, se propone el uso de optotriacs sincronizados con la red (cruce por cero) y la adición de tiempos de retardo de conexión equivalentes a medio ciclo de onda del periodo de la red, de esta manera, se busca permitir que los alternistores en uso puedan abrirse de forma natural y posteriormente proceder con la activación de los switches pertinentes.

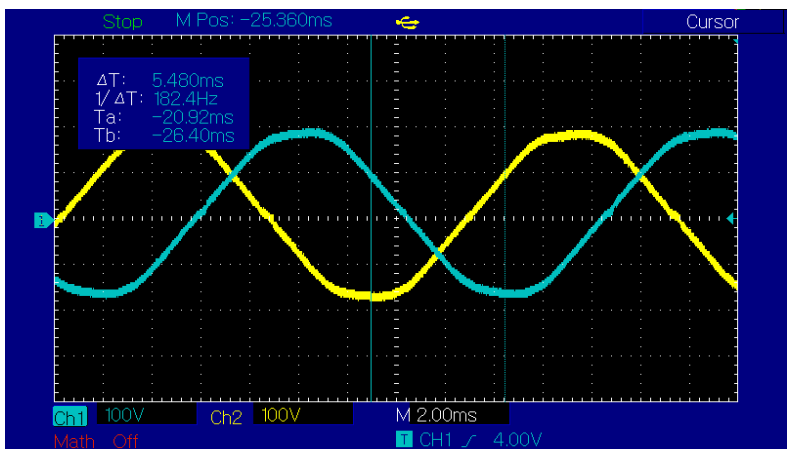

Figura 10. Desfase de $120^{\circ}$ entre las dos fuentes de energía utilizadas como entradas del IPS. Fuente. Elaboración propia.

A continuación, se presentan los escenarios de conmutación realizados:

\subsection{Intercambio en la fuente de alimentación de las cargas}

En la Tabla 2 se muestra el patrón de conexión utilizado, evidenciando que se realiza un intercambio en la fuente de alimentación de las cargas.

Tabla 2. Conmutación de las cargas en Figura 11.

\begin{tabular}{|c|c|c|}
\hline $\begin{array}{c}\text { Etapa de la } \\
\text { conmutación }\end{array}$ & Fuente 1 & Fuente 2 \\
\hline Antes & Carga 1 & Carga 2 \\
\hline Después & Carga 2 & Carga 1 \\
\hline
\end{tabular}

Fuente. Elaboración propia.

De acuerdo con la Tabla 2 y la Figura 11, se observa que antes de la señal de desconexión, cada una de las cargas se encuentra alimentada de forma independiente; es decir, la carga 1 está siendo alimentada por la fuente 1 (onda azul) y la carga 2 está conectada con la fuente 2 (onda amarilla).

Al momento de dar la señal de desconexión se observa que la conmutación no se realiza de forma inmediata,siendo necesario esperar al siguiente cruce por cero de las señales de tensión (la tensión y la corriente están en fase debido a que las cargas son resistivas) para que los switches del IPS se abran de forma natural. Para esto es importante dar un tiempo de espera de por lo menos medio ciclo de onda, con el fin de tener la certeza 
de que al momento de dar la señal de conexión todos los switches se encuentran abiertos y no existe riesgo de corto circuito.

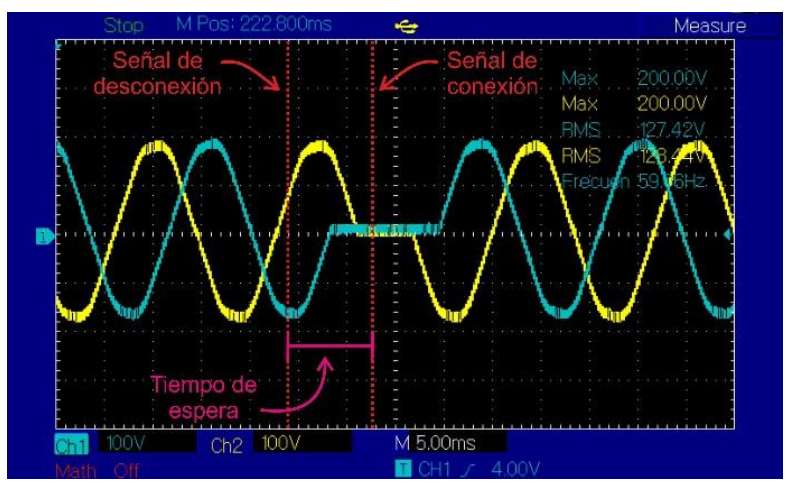

Figura 11. Tensión en las cargas al realizar la conmutación descrita en la Tabla I. Fuente. Elaboración propia.

\subsection{Cargas alimentadas por fuentes independientes a cargas alimentadas simultaneamente por la misma fuente}

En la Tabla 3. se muestra el patrón de conexión utilizado, mostrando que antes de la conmutación cada una de las cargas está alimentada por fuentes independientes y como después de la conmutación las dos cargas pasan a ser alimentadas de forma simultanea por una sola fuente.

Tabla 3 Conmutación de las cargas en Figura 12.

\begin{tabular}{|c|c|c|}
\hline $\begin{array}{c}\text { Etapa de la } \\
\text { conmutación }\end{array}$ & Fuente 1 & Fuente 2 \\
\hline Antes & Carga 2 & Carga 1 \\
\hline Después & -- & Carga 1 y 2 \\
\hline
\end{tabular}

Fuente. Elaboración propia.

En la Figura 12 se evidencia nuevamente la importancia del tiempo de espera entre la señal de desconexión y conexión de los aternistores, ya que debido al desfase de las fuentes, ante una conexión inadecuada de los switches se pueden producir corrientes muy elevadas o daños en los equipos.

Así mismo, este escenario de conexión es un caso que se puede dar en la práctica cuando una de las fuentes queda fuera de operación (por una falla, reparación o cualquier otro motivo); permitiendo que el sistema siga operando de forma normal, si las fuentes que aún se encuentran disponibles cuentan con la capacidad suficiente para cubrir la demanda de las cargas.

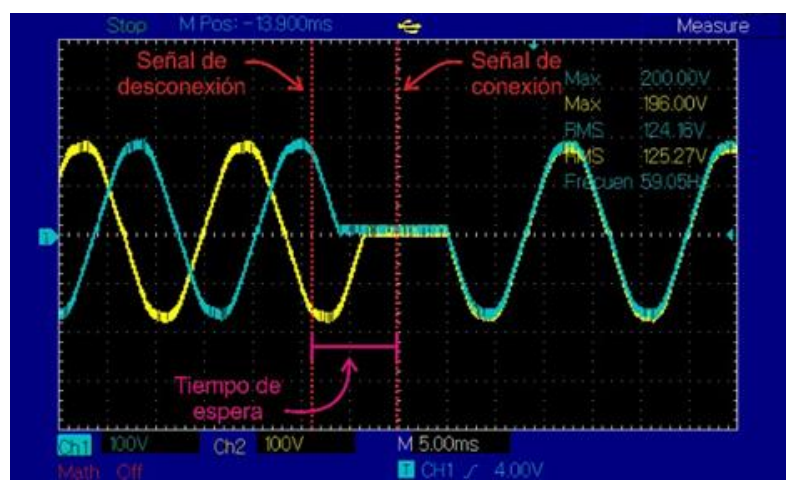

Figura 12. Esquema interno del conmutador inteligente de potencia. Fuente. Elaboración propia.

En caso de que una sola fuente no pueda suministrar la potencia suficiente para todo el sistema, se le puede dar prioridad a las cargas más importantes o delicadas.

\subsection{Cambio en la fuente de alimentación de las cargas}

Para este escenario de conexión es utilizado el patrón mostrado en la Tabla 4, permitiendo observar que antes de la conmutación las dos cargas son alimentadas de forma simultanea por la fuente uno y después de la conmutación las dos cargas pasan a ser alimentadas de forma simultanea por la fuente dos (Figura 13).

Tabla 4 Conmutación de las cargas en Figura 13.

\begin{tabular}{|c|c|c|}
\hline $\begin{array}{c}\text { Etapa de la } \\
\text { conmutación }\end{array}$ & Fuente 1 & Fuente 2 \\
\hline Antes & -- & Carga 1 y 2 \\
\hline Después & Carga 1 y 2 & -- \\
\hline
\end{tabular}

Fuente. Elaboración propia.

Con este caso de estudio se pretende mostrar la flexibilidad y las funcionalidades del sistema para responder ante diferentes condiciones de operación. El intercambio de las cargas de la fuente uno a la fuente dos es un escenario que en la práctica se puede dar cuando queremos migrar cargas de una fuente convencional a otra de características renovables (sistemas fotovoltaicos, turbinas eólicas, vehículo eléctrico, etc.), con el fin de buscar un ahorro económico.

De igual forma que en el ejemplo presentado en el numeral 8.2, el cambio en la fuente de alimentación de las cargas se puede dar cuando una de las fuentes queda fuera de operación y es necesario mantener en operación total o parcial el sistema. 


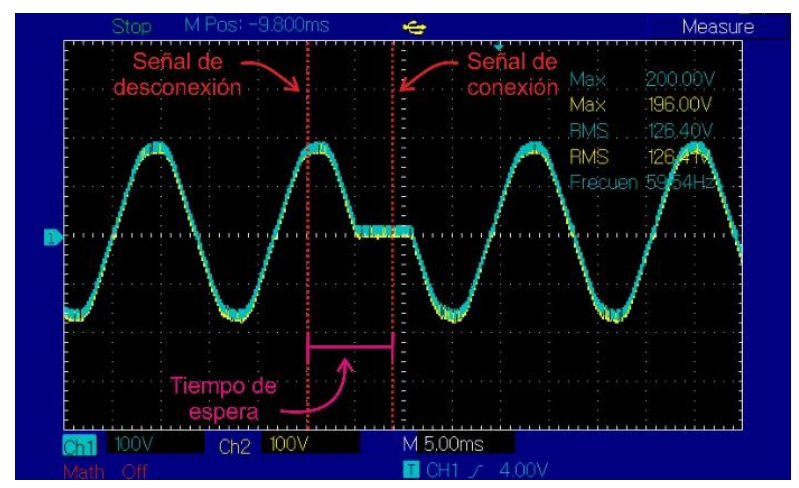

Figura 13. Esquema interno del conmutador inteligente de potencia. Fuente. Elaboración propia.

\subsection{Experimento 4}

El patrón de conexión utilizado para este caso de estudio es presentado en la Tabla 5, en la cual se muestra que antes de la conmutación las cargas son alimentadas de forma simultanea por la fuente uno y después de la conmutación, cada una de las cargas pasa a ser alimentada por una fuente independiente (Figura 14).

Tabla 5.Conmutación de las cargas en Figura 14.

\begin{tabular}{|c|c|c|}
\hline $\begin{array}{c}\text { Etapa de la } \\
\text { conmutación }\end{array}$ & Fuente 1 & Fuente 2 \\
\hline Antes & Carga 1 y 2 & -- \\
\hline Después & Carga 1 & Carga 2 \\
\hline
\end{tabular}

Fuente. Elaboración propia.

$\mathrm{Al}$ igual que en los casos anteriores, en la Figura 14 se observa que el tiempo de espera durante la conmutación es importante para evitar conexiones que puedan producir corrientes de corto circuito o daños en el sistema.

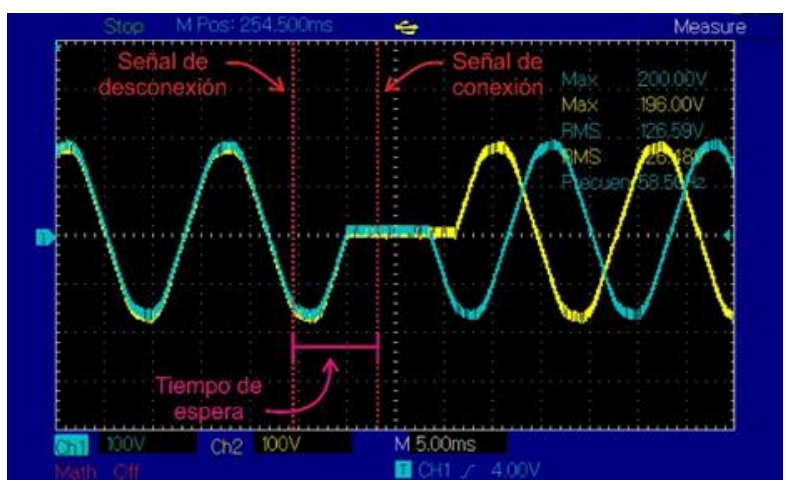

Figura 14. Esquema interno del conmutador inteligente de potencia. Fuente. Elaboración propia.
Este escenario de conexión se puede presentar en la práctica si una de las fuentes no está en capacidad de suministrar la totalidad de la potencia requerida por las cargas; para lo cual, es posible migrar parte del exceso de carga de la fuente uno a una segunda fuente con capacidad de suministro disponible.

\section{CONCLUSIONES}

Este artículo presenta la sub-medición por circuito y el conmutador inteligente de potencia como herramientas para la gestión energética en un hogar inteligente. Para esto se analiza el comportamiento de la tensión de las cargas conectadas al IPS ante diferentes escenarios de operación. De igual forma, debido al desfase de las fuentes de energía se propone el uso de optotriacs con cruce por cero y la adición de tiempos de espera en la conexión de los alternistores del IPS.

Los resultados experimentales muestran que el sistema propuesto tiene potencial como herramienta de gestión energética residencial, permitiendo controlar el flujo de energía entre las diferentes fuentes de energía (convencionales y renovables) y las cargas de una vivienda.

Igualmente, se evidencia que el uso de un tiempo de espera de medio ciclo de onda del periodo de la red permite que los alternistores tengan una desconexión (apertura) natural, lo cual es posible debido que durante el tiempo de espera la corriente llegue a su cruce por cero. Es importante resaltar que debido a que las cargas utilizadas son netamente resistivas, las señales de corriente y tensión se encuentran en fase. Sin embargo, al momento de usar cargas inductivas el tiempo de desconexión puede cambiar dependiendo de su factor de potencia.

Colombia ha realizado avances significativos en el contexto legal, normativo y reglamentario para la integración de las redes inteligentes en el sistema eléctrico nacional. Fomentando la investigación en temáticas como la gestión energética residencial y el IoT, permitiendo el desarrollo y la mejora del sistema energético. Adicionalmente, el progreso en esta temática es importante, ya que abre la puerta a diversos escenarios como la integración de la tarifa diferencial, la generación distribuida, la gestión de la demanda y en general fomenta la gestión y el uso racional y eficiente de la energía eléctrica.

La integración del conmutador inteligente de potencia en un hogar inteligente permite administrar los recursos energéticos de la vivienda y controlar el flujo de energía entre las diferentes fuentes y cada uno de las cargas, con el fin de reducir y optimizar el consumo de potencia, así 
como disminuir la compra de energía de la red pública. De esta manera, es posible contribuir con la reducción de la demanda de energía del país, apoyar el desarrollo de las redes inteligentes y fomentar el uso de fuentes renovables de energía eléctrica.

La sub-medición de energía eléctrica por circuito ramal es un componente importante del sistema de gestión que permite brindar información adicional a los usuarios en busca de mejorar hábitos de consumo y lograr una adecuada gestión energética. A partir de esta información, es necesario desarrollar herramientas intuitivas y amigables para presentar la información al usuario y permitir la monitorización y control de las diversas aplicaciones del hogar.

\section{RECOMENDACIONES}

Como trabajo futuro se espera disminuir el tiempo de espera en las conmutaciones, para lo cual se plantea la utilización de un sistema que permita detectar el desfase entre las fuentes por medio de las señales de tensión y corriente.

De la misma manera, se propone el uso de fuentes no convencionales de energía que se puedan sincronizar con la red, esto con el fin de poder inyectar energía a la red y/o para poder conectar simultáneamente dos o más fuentes y de esta forma satisfacer la demanda requerida por las cargas.

Igualmente, se sugiere la realización de pruebas con cargas inductivas para estudiar el comportamiento transitorio de la corriente al momento de las conexiones y desconexiones.

Finalmente, se sugiere la implementación un piloto en una vivienda familiar, con el fin de identificar el potencial de ahorro energético que se puede dar con la integración del IPS y la sub-medición por circuito.

\section{REFERENCIAS}

[1] M. R. Becerra, H. Mance, 'Cambio clima' tico: lo que esta' en juego". Foro Nacional Ambiental, 2009.

[2] University of Cambridge and Worl Energy Council, "Cambio Climático: Implicaciones para el sector energético, hallazgos claves del quinto informe de evaluación del grupo intergubernamental de expertos sobre el cambio climático."

[3] S. S. S. R. Depuru, L. Wang, and V. Devabhaktuni, "Electricity theft: Overview, issues, prevention and a smart meter based approach to control theft", Energy Policy, vol. 39, no. 2, pp. 1007-1015, Feb. 2011.
[4] Hassan Farhangi, "The Path of the Smart Grid", IEEE power \& energy magazine, no. february, 2010.ogan,

[5] Y. Kitamori, R. Takahashi, T. Hikihara, "Circuit Switching by Power Routers in Power Distribution Network", IEEE 2nd Global Conference on Consumer Electronics (GCCE), 2013.

[6] T. Takuno, Y. Kitamori, R. Takahashi, T. Hikihara, "AC Power Routing System in Home Based on Demand and Supply Utilizing Distributed Power Sources", Energies, vol. 4, no. 5, pp. 717-726, 2011.

[7] Toyoda J. and Saitoh H, "Proposal of an OpenElectric-Energy- Network (OEEN) to Realize Cooperative Operations of IOU and IPP”. In Proceedings of the 1998 International Conference on Energy Management and Power Delivery (EMPD), Singapore, 5 March 1998; Volume 1, pp. 218-222.

[8] R. Takahashi, Y. Kitamori and T. Hikihara, "AC Power Local Network with Multiple Power Routers", Energies, vol. 12, no. 5, pp. 6293-6303,2013.

[9] K. Ehrhardt-martinez, J. A. S. Laitner, and K. A. Donnelly, "Beyond the Meter: Enabling Better Home Energy”, no. 2010. Elsevier, 2011, pp. 273-303.

[10] T. Ueno, R. Inada, S. Osame, and K. Tsuji, "Effectiveness of display- ing energy consumption data in residential houses Analysis on how the residents respond", Panels of ECEEE, pp. 1289 - 1299, 2005.

[11] R. Lutolf, "Smart Home Concept and The Integration of Energy Meters Into a Home Based System", Landis \& Gyr Energy Management Corp., Switzerland.

[12] M. A. A. Pedrasa, S. Member, T. D. Spooner, and I. F. Macgill, "Coordinated Scheduling of Residential Distributed Energy Resources to Optimize Smart Home Energy Services”, Energy, vol 1, no 2, pp 134 -143, 2010.

[13] C. Nahr, "Smart Home with Solar Electricity", Disponible en: http://en.smasunny.com/2012/08/07/smart- home-with- solarelectricity/

[14] D. Menga , D. Durand , B. Marhic , L. Delahoche , and C. Loge, "The Smart Home Concept: our immediate," IEEE, pp. 23-28, dec 2006.

[15] El Congreso de Colombia, "Ley 1715 de 2014", Ministerio de Hacienda y Cre'dito $\mathrm{Pu}^{\prime}$ blico, de de Minas 
y Energ'ia, de Ambiente y Desarrollo Sostenible y COLCIENCIAS, 2014.

[16] Ministerio de Vivienda, Ciudad y Territorio, "Decreto 1285 de 2015", 2015.

[17] Ministerio de Vivienda, Ciudad y Territorio, "Resolucio' n 0549 de 2015”, 2015.

[18] El Congreso de Colombia, "LEY 697 DE 2001 - Ley URE," Ministerio de Minas y Energ'1a, vol. 2001, no. octubre 3, pp. 3-6, 2001.

[19] O. PRIAS CAICEDO, "Informe final PROURE," Ministerio de Minas y Energ' 1a, pp. 16-86, 2010.

[20] El Presidente De La Repu' blica De Colombia, "DECRETO 3683 DE 2003," Ministerio de Minas y Energ'ia, vol. 2003, no. 45, 2003.

[21] El Presidente De La Repu' blica De Colombia, "DECRETO 2501 DE 2007," Ministerios de Minas y Energía, de Comercio, Industria y Turismo y de Ambiente, Vivienda y Desarrollo Territorial., pp. 2-3, 2007.

[22] El Presidente De La Repu' blica De Colombia, "DECRETO 3450 DE 2008," Ministerio de Minas y Energ' 1 a, 2008.

[23] Ministerio de Minas y Energía, "Resolución 180919 de 2010," 2010. [24] Unidad de Planeación Minero Energética - UPME, "Programa Colombiano de Normalización, Acreditación, Certificación y Etiquetado de Equipos de Uso Final de Energ'ra- CONOCE," UPME.

[25] International Organization for Standardization ISO, "ISO 50001 Gestión de la Energía," 2011.

[26] Ministerio de Minas y Energía, "Resolución 038 de 2014," 2014.

[27] Instituto Colombiano de Normas Técnicas y Certificación, ICONTEC, "NTC 6079 - Requisitos para sistemas de infraestructura de medición avanzada (AMI) en redes de distribución de energía eléctrica"

[28] T. Takuno, M. Koyama and T. Hikihara, "In-home power distribution systems by circuit switching and power packet dispatching", First IEEE International Conference on Smart Grid Communications (SmartGridComm), pp. 427-430, Oct. 2010.

[29] M. He, E. Reutzel, X. Jiang, R. Katz, S. Sanders, D. Culler, and K. Lutz, "An architecture for local energy generation, distribution, and sharing," in IEEE Energy 2030, Atlanta, Georgia, USA, November 2008.

[30] G. Lu, D. De and W. Z. Song, "SmartGridLab A Laboratory- Based Smart Grid Testbed", First IEEE International Conference on Smart Grid Communications (SmartGridComm), pp. 143 - 148, Oct. 2010.

[31] Jose Manuel Rodríguez, "Submetering como pieza clave en la gestión eficiente de la energia," 2 congreso eficiencia energetica eléctrica, 2012. 